\title{
Bone marrow derived stem cells facilitate axonal regeneration in a rat model of 2,5-hexanedione- induced neuropathy via miRNA-dependent and independent routes
}

\section{Enjun Zuo}

Dalian Medical University

Shuhai Hu

Dalian Medical University

Cong Zhang

Dalian Medical University

Xiaoxia Shi

Dalian Medical University

\section{Zhaofei Yang}

First Affiliated Hospital of Dalian Medical University

Shuangyue Li

Dalian Medical University

\section{Qiufang Bai}

Affiliated Zhongshan Hospital of Dalian University

\section{Guanyu Gong}

Affiliated Zhongshan Hospital of Dalian University https://orcid.org/0000-0002-5791-0916

\section{Ruoyu Wang}

Affiliated Zhongshan Hospital of Dalian University

Fengyuan Piao ( $\nabla$ piao_fy_dy@163.com )

Affiliated Zhongshan Hospital of Dalian University

\section{Research}

Keywords: N-hexane, 2,5-hexanedine, Peripheral neuropathy, BMSCs, Axon regeneration, NGF, Let-7 miRNA, Schwann cells

Posted Date: August 27th, 2021

DOl: https://doi.org/10.21203/rs.3.rs-832403/v1 
License: (c) (i) This work is licensed under a Creative Commons Attribution 4.0 International License. Read Full License 


\section{Abstract \\ Background}

Peripheral polyneuropathies are clinically difficult situations and can be caused by exposure to occupational hazardous materials, such as $\mathrm{N}$-hexane, which is still being used in developing countries. Autologous transplantation with bone marrow-mesenchymal stem cells (BMSCs) could be a promising therapeutical approach but its effect on toxic chemical-induced neuropathy remains undetermined. The present study sought to understand whether BMSCs could improve axon regeneration in Hexane-caused polyneuropathies and attempted to understand the associated molecular mechanisms.

\section{Methods}

A rat model of 2,5-hexanedine (HD)-induced polyneuropathy was established by intraperitoneally injection of $400 \mathrm{mg} / \mathrm{kg} /$ day HD, 5 times/week for 5 weeks. After the development of characteristic phenotypes, rats were given a single bolus tail-vein injection of BMSCs $\left(5 \times 10^{7}\right.$ cells $\left./ \mathrm{kg}\right)$ and followed-up for 5 weeks. Sciatic nerves of HD/HD + BMSCs treated rats, as a representative tissue for peripheral nerve, were analyzed using electron microscopy, immunohistochemistry, western blotting and RNA sequencing. To further dissect the underlying mechanism, cultured DRG neurons +/-Schwann cells were challenged with $\mathrm{HD} / \mathrm{BMSC}$-derived conditional medium (BMSC-CM), in the presence or absence of NGF/Akt/mTOR inhibitors, recombinant NGF, miRNA mimics or miRNA inhibitors and further tested for changes in cellular and molecular levels.

\section{Results}

BMSCs engrafting significantly improved motor function recovery. Accelerated axon regeneration in sciatic nerves was evidenced by transmission electron microscopy, GAP43 western blotting and MBP/SMI312 immunostaining. Increased NGF expression and signaling activation was evidenced in response to BMSC/BMSC-CM treatment. In cultured cells, BMSC-CM's effect was abolished by anti-NGF antibody or TrkA inhibitor K252a but reinforced by recombinant NGF. The activation of PI3K-Akt-mTORCREB signaling was observed and Akt and mTOR inhibitors were shown to attenuate BMSCS-promoted axon regeneration. While NGF was shown to be secreted by BMSCs in culture medium, miRNA array suggested certain miRNAs might also participate to regulate NGF expression. Three let-7 family miRNAs, including let-7a-5p, let-7d-5p and let-7e-5p, and 69 other miRNAs were found to be differentially expressed in HD and HD/BMSC groups. The regulatory effect of let-7e-5p on NGF expression was examined using miRNA mimics and inhibitors.

\section{Conclusions}


Together, our study demonstrated that BMSC transplantation significantly improve axon regeneration in hexane-induced polyneuropathy by activating NGF-PI3K-Akt-mTOR-CREB signaling via two independent mechanisms.

\section{Introduction}

Peripheral neuropathy (PN) refers to a variety of neurological conditions involving damage to the peripheral nervous system, commonly caused by trauma, diabetes, microvasculopathy and exposure to certain toxic chemicals. N-hexane, with its active biometabolite 2,5-hexanedine (HD), is a hazardous chemical widely used in industry and it is still being used in some developing countries [1]. Hexane intoxication presents with classic clinical manifestations of peripheral neuropathies, including numbness and tingling of the extremities and progressive weakness of limb muscle [2]. Pathological observations of the peripheral nerves showed axon swelling and atrophy, segmental demyelination and Wallerian degeneration, similar in human patient biopsies and experiment animals [3-5].

Once being damaged, the peripheral nerves, formed by axons of terminally differentiated neurons and glial cells, are well-known to process with a slow and limited recovery and serious damage may result in permanent debilitation or disability. Therefore, medical interventions assisting nerve regrowth are needed. However, to date, the best treatment remains to be prompt surgical repair for traumatic lesion but no effective methods are available for peripheral neuropathy induced by toxins, diabetes and vascular diseases [6].

Mesenchymal stem cells (MSCs), including bone marrow-derived MSCs (BMSCs), are adult stem cells in connective tissue with strong self-renewing and differentiation potency [7]. MSCs transplantation has been regarded a promising new therapeutical approach in PN management [6]. Several studies found that transplantation of mesenchymal stem cells (MSCs) promoted axonal regeneration and enhanced hindlimb motor function recovery in rats with contusive [8-10] or transected spinal cord injury (SCI) [11]. Some other investigators found that BMSCs could increase retinal ganglion cell survival and promote axonal regeneration after optic nerve injury in rodents [12-14]. Moreover, transplantation of adiposederived MSCs was found to promote axon myelination and regrowth and ameliorate muscle atrophy in a mouse model of sciatic nerve denervation[15]. In these pre-clinical studies, MSCs transplantation has been shown to promote nerve regeneration in both peripheral and central system. Nonetheless, it remains to be determined whether BMSCs transplantation could ameliorate PN caused by toxic chemicals such as $\mathrm{N}$-hexane and through which mechanism(s) BMSC exert its effect. To this end, our research group conducted several studies to improve our answer for this particular question.

Mechanistically, the beneficial effect of BMSCs on nerve regeneration has been associated with multiple soluble factors, such as cytokines and neurotrophic factors (NTFs), that are released from MSCs to support nerve regeneration, axon remyelination, and protecting neurons[16-18]. In our early report, conditional medium from culture BMSCs was shown to protect VSC4.1 motor neurons against 2,5-HDinduced damage by secreting nerve growth factor (NGF) and activating the neurotrophic tyrosine kinase 
receptor type 1 (TrkA)[19]. Others also showed that axonal elongation in chicken embryo dorsal root ganglia (DRG) responded dose-dependently to NGF[20].

In the present study, the neuroprotective effect of BMSCs was firstly evaluated in a rat model of HDinduced PN. Neurobehavior test, nerve conduction measurement and histopathological analysis focused on the changes in the sciatic nerve. Next, to further dissect the molecular mechanisms associated with BMSCs' effect, dorsal root ganglion (DRG) neurons were cultured in vitro and treated with HD and with or without BMSC-derived conditional medium (BMSC-CM). Those important signaling molecules involved in BMSC's biological function were measured and further studied using selective inhibitors. Our findings suggest that BMSC transplantation may be an effective strategy to treat HD-induced PN and revealed some fundamental mechanisms underlying this process.

\section{Material And Methods}

\section{Animal experiments}

All animal experiment protocols were approved by the Ethical Committee of Dalian Medical University, China (approval number: CXK (Liao) 2015 - 2003) and performed following the Institutional Animal Care and Use Committees (IACUC) of Dalian Medical University and following the international guidelines for the animal care and use (ARRIVE guidelines and NIH guidelines). The HD-intoxication experiment was as being described in our earlier publication [21]. Briefly, adult male Sprague Dawley rats $(200-230 \mathrm{~g}$ body weight) were maintained in the Experimental Animal Center of Dalian Medical University. Routine husbandry procedure was provided with $22^{\circ} \mathrm{C}$ room temperature, $50 \%$ relative humidity, a $12 \mathrm{~h}$ light-dark cycle and supply of food and drinking water ad libitum. To induce HD-mediated neurotoxicity, rats were intraperitoneally injected with HD (Solarbio, China), $400 \mathrm{mg} / \mathrm{kg} /$ day, five times per week, for 5 consecutive weeks. After the development of characteristic phenotypes of peripheral nerve degeneration, rats were engrafted with $5 \times 10^{7}$ cells/kg BMSCs (in a single bolus dose) [22] by tail vein injection and observed to follow-up functional recovery for 5 weeks until necropsy. The control rats received no BMSCs treatment but only normal saline (NS). At the end of week 10, rats were necropsied and the sciatic nerves were quickly dissected, soaked in fixation solution or snap-frozen in liquid nitrogen for further analyses.

\section{Neurobehavioral and nerve electrophysiological tests}

To assess the phenotypical effect of axon degeneration/regeneration, gait score test, distal latency test and motor nerve conduction velocity test were carried out[23, 24]. A well-trained, test-blinded neurologist who was not involved in the project was asked to perform the measurements.

A gait score was assigned from 1 to 4 , with 1 = normal, unaffected gait; 2 = slightly affected gait (tip-toe walking, slight ataxia, and hindlimb weakness); 3 = moderately affected gait (obvious movement abnormalities characterized by dropped hocks and tail dragging); 4 = severely affected gait (frank hindlimb weakness and inability to rear). Three successive measurements were taken and averaged for each animal [25]. 
The distal latency and conduction velocity were measured once a week, using a method published in literature [26]. The rats were anesthetized with ether and placed in a self-made rat fixation at the prone position. Three testing points, A, B and C, were marked at $3 \mathrm{~cm}, 10 \mathrm{~cm}$ and $15 \mathrm{~cm}$ from the base of the tail, respectively. Stimulation electrode was placed at Point $A$, and recording electrodes were placed at Points $B$ and $C$. The nerve conduction velocities between $A$ and $B$ and between $A$ and $C$ were recorded. One hundred impulses were recorded and summated to calculate the conduction velocity: motor nerve conduction velocity $(\mathrm{MCV})=$ Length $B C$ / Latency $B C$ (Length $B C$ is the distance between $B$ and $C$, Latency $B C$ is the latency time between $B$ and $C$ ). Distal latency was expressed using the latency $B C$ time.

\section{Electron microscope (SEM) analysis}

Sciatic nerves were immersed in $2 \%$ glutaraldehyde overnight at $4^{\circ} \mathrm{C}$, rinsed in PBS, fixed with $1 \%$ acetic acid for 3hrs, subjected to gradient dehydration, embedded and sliced with an ultramicrotome[27-29]. The slides were then stained with uranyl acetate and lead citrate and observed under a transmission electron microscope (H-7500, Hitachi, Japan).

\section{BMSCs isolation and culture}

BMSCs were prepared as being described [30]. Briefly, Sprague Dawley rats (5-6 weeks old, $60-90 \mathrm{~g}$, Laboratory Animal center, Dalian Medical University, China) were euthanatized and bone marrow was flushed from the femur and tibia bones with PBS. A mixture of single-cells was obtained by passing the cells through a sieve, seeded in Dulbecco's modified eagle medium-low glucose (Gibco, USA) supplemented with $10 \%$ fetal bovine serum (FBS, HyClone, USA), $100 \mathrm{U} / \mathrm{mL}$ penicillin and $100 \mu \mathrm{g} / \mathrm{mL}$ streptomycin (Beyotime, China) and culture at $37^{\circ} \mathrm{C}$ with $95 \%$ humidified air. Twenty-four hours later, the medium was replaced. The cells were cultured and used in in vivo or in vitro experiments within 5 passages. To validate the correct harvest and culture of these BMSCs, they were immunocytochemically stained using neuron specific enolase (NSE) antibody 24,48 and $72 \mathrm{~h}$ after seeding. Adipogenic and osteogenic differentiation of BMSCs was evaluated by specific media Oil Red $\mathrm{O}$ and Alizarin Red S staining (Cyagen, China) [31].

\section{Preparation of BMSCs-derived conditional Medium (BMSC- CM)}

BMSC-CM was prepared following an established method[32]. Briefly, confluent BMSCs were washed, supplemented with fresh DMEM-10\% FBS and cultured for $24 \mathrm{~h}$. The culture media were collected, centrifuged at $200 \mathrm{~g}$ for 10 minutes at $4^{\circ} \mathrm{C}$, applied to a filtration column (Millipore, Billerica, MA, USA), centrifuged at $3,500 \mathrm{~g}$ for 45 minutes at $4^{\circ} \mathrm{C}$, desalted and filter-sterilized.

\section{Culture of primary Schwann cells and DRG neurons}

To harvest DRG neurons, 2 day-old SD rats were sacrificed and lumbar dorsal root ganglia were dissected under a $16 x$ anatomical microscope. The tissue was washed, cut and digested using $0.5 \%$ Type II Collagenase (Sigma, USA) for 45 minutes at $37^{\circ} \mathrm{C}$ and then with $0.25 \%$ trypsin for 10 minutes at $37^{\circ} \mathrm{C}$. The dissociated DRG neurons were centrifuged, resuspended, filtered, and cultured with D-MEM/F-12 
supplemented with 10\% FBS, 2\% B-27 (Gibco, USA), $0.25 \mathrm{mg} / \mathrm{ml}$ insulin (Sigma, USA), $0.1 \mathrm{mg} / \mathrm{ml}$ Lglutamine (Sigma), $100 \mathrm{U} / \mathrm{ml}$ penicillin and $100 \mathrm{mg} / \mathrm{ml}$ streptomycin at $37{ }^{\circ} \mathrm{C}$ with $5 \% \mathrm{CO} 2$.

To harvest Schwann cells, 5-day-old SD rats were sacrificed and sciatic nerves were cut and digested with $0.125 \%$ trypsin and $0.03 \%$ type IV collagenase (Beyotime, China) for 30-40 min and centrifuged at 1,500 $\mathrm{g}$ for $5 \mathrm{~min}$. The Schwann cells were cultured in DMEM with $10 \%$ FBS and with $1 \mathrm{uM}$ cytarabine at $37^{\circ} \mathrm{C}$, $5 \% \mathrm{CO} 2$ for $24 \mathrm{~h}$. For co-culture, Schwann cells were seeded on a density of $1 \times 10^{6} / \mathrm{mL}$ and DRG neurons were seeded on $5 \times 10^{4} / \mathrm{mL}$ onto two poly-L-lysine-coated $0.5 \times 0.5 \mathrm{~cm}$ coverslips, respectively. After 3-4 days of culture, the two coverslips with Schwann cells and DRG neurons were placed together with a distance of $2 \mathrm{~mm}$ and continued to grow for 3 days.

\section{Cell transfection}

Lipofectamine 3000 transfection reagent (Invitrogen, USA) was used to deliver NGF siRNA, scramble control siRNA, let-7e-5p mimics, let-7e-5p anti-miRNA and control miRNA into cells. All the siRNAs and miRNAs were purchased for RiboBio (China).

\section{Treatment of DRG neurons with BMSC-CM, NGF, anti-NGF, and $\mathrm{K} 252 \mathrm{a}$}

DRG neurons were cultured and added with HD (8 mM), HD (8 mM) plus 20\% (v/v) BMSC-CM, HD (8 mM) plus anti-NGF (1:500) plus 20\% BMSC-CM, HD (8 mM) plus non-specific immunoglobulin (1:500) plus 20 $\%$ BMSC-CM, HD (8 mM) plus recombinant NGF $(200 \mu \mathrm{g} / \mathrm{mL})$, or HD $(8 \mathrm{mM})$ plus K252a $(200 \mathrm{nM})$ plus 20 $\%$ BMSC-CM. The cells were grown for additional $12 \mathrm{hrs}$ before harvest.

\section{ELISA analysis of NGF}

The concentrations of NGF in sciatic nerve tissue, BMSC-CM and cell culture medium were detected using an enzyme linked immunosorbent assay (ELISA) kit (USCN, USA) following manufacturer's instruction. Medium of cells were collected and filtered through a $0.22 \mu \mathrm{m}$ filter (Millipore) for removing cell debris. Each sample were measured three times using a spectrophotometer with absorbance at $490 \mathrm{~nm}$ wavelength.

\section{Western Blot}

Tissues and cells were homogenized using ice-cold RIPA buffer (Beyotime, China). Protein lysates were separated on SDS-PAGE gel and transferred to PVDF membrane (Millipore, France). The membrane was blocked and incubated with primary antibodies as following: NGF (Cell Signaling Technology, USA), TrkA, p-TrkA, Akt, p-Akt, mTOR, p-mTOR, CREB, p-CREB, GAP-43, GAPDH (Abcam, USA), and $\beta$-actin (ZS-Bio, China). The membranes were incubated with horseradish peroxidase-conjugated secondary antibody (Sigma, USA) and immunoreactivity was visualized enhanced chemoluminescence (Beyotime, China). For quantification, each sample was subjected to three independent experiments $(n=3)$ with triplicate loading per experiment.

\section{Immunofluorescence}


For immunofluorescence, frozen tissue sections $(10 \mu \mathrm{m})$ or cold methanol-fixed DRG neurons were blocked with $10 \%$ donkey serum, incubated with either anti-MBP (Cell signaling, USA) or anti-MAP2 antibody (Cell signaling, USA) overnight at $4^{\circ} \mathrm{C}$ and fluorophore-conjugated secondary antibody, then incubated with anti-SMI312 (Cell signaling) or anti-GAP-43 antibody (Cell signaling) and secondary antibody. The stained slides were observed and imaged under a confocal microscope (Olympus, Japan). Image analysis was performed using Image-Pro Plus.

\section{Microarray analysis of miRNAs}

The expression profile of miRNAs in sciatic nerves of rats receiving no treatment (control), HD, and HD + BMSC was investigated using a miRNA microarray chip (KangChen Bio-Tec, China). The differential expression of miRNAs was analyzed using fold change $(\mathrm{FC})>2$ and $p$-value $<0.05$, respectively, in comparison between control and HD groups and between HD and HD + BMSC groups. Heatmap and clustering from sequencing were used to show the differential expression of miRNAs.

\section{Real Time-PCR}

Total RNA from sciatic nerve tissues and culture cells was extracted using RNAiso Plus (Takara, Japan). Real Time PCR was performed to analyze expressional change of Let-7 miRNAs and NGF mRNA using

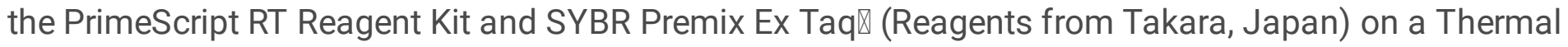
Cycle Dice Real-Time system. The Bulge-Loop miRNA primers for let-7 miRNAs and U6 miRNA were from RiboBio (China). The primers (NGF Forward primer 5'-3': TGC CCC TGC TGA ACC AA, Reverse primer 5'-3': GCT TGC TCC TGT GAG TCC TGT; GAPDH Forward primer 5'-3': GGC ACA GTC AAG GCT GAG AAT G; Reverse primer 5'-3': ATG GTG GTG AAG ACG CCA GTA) were also from RiboBio (China). All reactions were run in triplicate. The relative expression was calculated using the comparative $2^{-\Delta \Delta \mathrm{Ct}}$ method.

\section{Statistical Analysis}

All results were expressed as mean $\pm S D$, and the statistical analysis was performed with one-way analysis of variance (ANOVA), followed by LSD test, which was performed using SPSS 13.0 statistical software. The p-values less than 0.05 were considered to be significant.

\section{Results}

\section{BMSC Transplantation alleviated HD-induced peripheral neuropathy in rats}

In order to determine whether BMSC transplantation have therapeutical potential in peripheral neuropathy caused by HD intoxication, a rat model was established, as being illustrated in Fig. 1A. Peripheral neuropathy was induced by intraperitoneal injection of HD (400 mg/kg/day, 5 times/week) for 5 weeks. Next, a bolus of BMSCs or normal saline (control) was administrated by tail vein injection $\left(5 \times 10^{7} \mathrm{cells} / \mathrm{kg}\right.$ body weight) and rats were followed-up for another 5 weeks to observe recovery. The nature of BMSCs was characterized and shown in Supplemental Fig. 1. 
As displayed in Fig. 1B, those rats receiving HD injection presented with characteristic phenotypes including waddling gait, weakness of hind limbs, muscle wasting and loss of weight. These symptoms progressively deteriorated and became apparent after 5 weeks. BMSC transplantation resulted in markedly improved motor function and muscle regain, when comparing Fig. 1B HD + BMSCs group with HD + NS group. Quantitative analysis of gait score and neurophysiological evaluation of motor nerve conduction (distal latency and conduction velocity) all demonstrated restored peripheral nerve function (Fig. 1C-E).

Next, transmission electron microscopy was used to observe the cellular changes in the peripheral nerves after HD intoxication and BMSC transplantation, using the sciatic nerve as a prototype for our study. As shown in Fig. 1F, the nerves of unintoxicated rats (control group or BMSCs group) featured with regular oval-shaped axons surrounded with smooth myelin sheaths (yellow arrows). The nerves of HDintoxicated rats (HD group or HD + NS group) showed various features of neurodegeneration, including deformity in shapes, decreased fiber diameter and vacuole aggregation in axons (red arrows and boxes). In comparison, with BMSC treatment, the nerves of HD + BMSC group presented with less vacuole aggregation, more intact structure and larger axon calibers, suggesting that nerve regeneration had occurred in response to BMSC engrafting after HD intoxication. The nerve regrowth was also examined by western blot using anti-GAP-43 antibody and by immunohistochemistry using SMI312 (labelling axon) and MBP (labelling myelin sheath) antibodies, respectively. As shown in Fig. 1G-I, GAP-43 protein abundancy, that was used to indicate neurogenesis, was reduced after HD intoxication and recovered after BMSC treatment. SMI312 immunostaining also suggested damaged axons after HD treatment and axon regrowth after BMSC engrafting.

BMSC Transplantation increased NGF level in vivo and in vitro

In literatures, BMSCs has been suggested capable of secreting NGF, an important neuroprotective factor functioning in neurogenesis $[33,34]$. To determine whether NGF had participated in the BMSC-elicited protection in our rat model of HD neurotoxicity, NGF concentrations in serum and sciatic nerves were measured using ELISA assay. As shown in Fig. 2A-B, NGF level was found significantly decreased after $\mathrm{HD}$ treatment and increased after BMSC transplantation. The protein expression levels of NGF, TrkA (NGF receptor) and pTrkA (TrkA activation) also suggested activation of NGF-TrkA by BMSCs (Fig. 2C-D).

Next, aiming to explore the responsible mechanisms underlying BMSCs' effect on axon regeneration, Schwann cells and DRG neurons were co-cultured and treated with HD and with/without BMSC-derived conditional medium (BMSC-CM, $20 \% \mathrm{v} / \mathrm{v}$ ) to mimic the challenges in vivo (Fig. 2E). NGF was evidenced to be present in BMSC-CM using ELISA assay (supplemental Fig. 2). The protein expressions of NGF, TrkA and pTrkA in cultured cells were immunoblotted and showed decreased levels in response to HD and increased levels after culturing with BMSC-CM (Fig. 2F-G). Together, these data indicated that NGF secretion and signaling activation might play a key role in BMSCs' neuroregenerative effect.

\section{NGF signaling participated in BMSCs-promoted axon regeneration}


In order to understand how important NGF is for BMSCs-promoted axon regeneration, DRG neurons were treated with HD $(8 \mathrm{mM})$ for $24 \mathrm{~h}$ and then with recombinant NGF $(200 \mu \mathrm{g} / \mathrm{mL})$, BMSC-CM, BMSC-CM added with anti-NGF monoclonal antibody $(10 \mu \mathrm{M})$ and BMSC-CM added with control non-specific immunoglobulins (as diagramed in Fig. 3A). Western blot was used to assess GAP-43 protein expression (neurite growth specific marker) (Fig. 3B) and immunofluorescent co-staining of MAP2 (neuron-specific marker) and GAP-43 was used to observe the morphological changes (Fig. 3C). The length of DRG neurite was measured in cells stained with GAP-43 and compared among different groups (Fig. 3D). The results suggested that axon regeneration was induced equivalently in response to either BMSC-CM or recombinant NGF, but such an effect was diminished in the presence of NGF neutralizing antibody.

Next, activation of NGF signaling was experimentally blocked using the TrkA inhibitor k252a (200mM). HD-treated cells were cultured with BMSC-CM in the presence or absence of k252a (diagramed in Fig. 3E). The protein expression of GAP-43, MAP2-GAP43 co-immunostaining and the neurite length measurement all suggested that axon regrowth was inhibited after addition of k252a (Fig. 3F-H). Together, our results suggested that NGF signaling activation is indispensable for axonal regeneration induced by BMSCs.

\section{NGF activated PI3K-Akt-mTOR-CREB pathway to promote axon regeneration}

Next, our work sought to explore the signaling pathway downstream to NGF/TrkA activation. The protein expression levels of total and activated (phosphorylated) Akt, mTOR and CREB signal transducers were immunoblotted and compared among different treatment groups (Fig. 4). The results suggested that the activation of Akt (p-Akt/total Akt), mTOR (p-mTOR /total mTOR) and CREB (p-CREB /total CREB) signals was significantly decreased by HD treatment and increased by BMSC transplantation or BMSC-CM treatment, in rat sciatic nerves (Fig. 4A-C) and in cultured DRG neurons (Fig. 4D-F).

The impacts of PI3K-Akt-mTOR-CREB activation on axon regeneration were exemplified by addition of various signal inhibitors including k252a (TrkA inhibitor), MK-2206 (Akt inhibitor) and Rapamycin (mTOR inhibitor) to HD/BMSC-CM-treated DRG neurons (Fig. 5A). The effectiveness of these inhibitors was validated, as being suggested by the decreased p-Akt level by k252a (Fig. 5B), decreased p-mTOR level by MK2206 (Fig. 5C) and decreased pCREB level by Rapamycin (Fig. 5D), respectively. The blockade of PI3KAkt-mTOR pathway was, thereafter, found to abort BMSC-CM's ability to induce axon regeneration. The changes of GAP-43 protein expression (Fig. 5E), neurite outgrowth (Fig. 5F) and neurite length (Fig. 5G) by BMSC-CM were all shown to be cancelled after addition of these inhibitors. Together, our results demonstrated that the PI3K-Akt-mTOR-CREB signaling molecules are the key downstream targets of NGF/TrkA activation during BMSC-induced axonal regeneration.

\section{NGF expression was modulated by let-7 miRNAs in Schwann cells}


MicroRNAs (miRNAs) are a class of non-coding RNAs that regulate gene expressions by promoting degradation or inhibiting protein translation of target mRNAs, therefore participating in many biological

processes. An earlier study has reported that the expression of NGF was negatively correlated with certain let-7 family miRNAs after sciatic nerve injury [35]. In order to explain the changed NGF protein expression after BMSC treatment, we were interested to understand whether BMSCs could promote axon regeneration via direct modulation of NGF mRNA (and protein) expression by interfering with the miRNAregulatory pathways. To this end, miRNA microarray was conducted to analyze the expressions of miRNAs in the sciatic nerves of untreated, HD-treated and HD + BMSC transplantation-treated rats.

As shown in Fig. 6A, differential expressions of multiple let-7 family miRNAs were observed among treatment groups. The results were validated using qPCR (Fig. 6B-E). The expressions of three let-7 members, namely let-7a-5p, let-7d-5p and let-7e-5p, were significantly increased in sciatic nerves of rats receiving $\mathrm{HD}$ treatment and decreased after transplantation with BMSCs (Fig. 6B-D). In addition, let-7d-3p was found significantly increased by HD and slightly decreased by BMSCs (Fig. 6E). These results suggested that aberrant expression of let-7 miRNAs happened after HD intoxication and BMSCs transplantation had certain modulatory effects on let-7 miRNA expression.

While literatures suggested that the Schwann cells are the main secretory resource of NGF[36], the present study also investigated the expression profiles of aforementioned miRNAs in Schwann cells receiving HD +/- BMSC-CM treatment (Fig. 6F). As shown in Fig. 6G-J, the change of let-7 member expressions in Schwann cells demonstrated the same tendency as the findings in vivo. This suggested that Schwann cells could be the specific recipient of let-7 miRNAs in response to HD and BMSC transplantation.

\section{Regulation of NGF expression by let-7 associated with axon regeneration}

Next, our work testified whether the expressional change of let-7 miRNAs would cause changes in NGF expression and axon regeneration. In Li et al's publication, let-7d miRNA was suggested capable of binding to the 3'-untranslated region (3'UTR) of NGF mRNA[35]. Herein our study identified that the miRNA let-7e-5p was the most significantly changed let-7 family members comparing the HD + BMSC group with the HD group (Fig. 6) and therefore let-7e-5p was selected as the representative miRNA in the continual experiments.

By matching the let-7e-5p miRNA sequence with the NGF mRNA sequence, the presence of let-7e-5p seed sequence was identified within the $3^{\prime} U T R$ of NGF gene (Fig. 7A). Then let-7e-5p mimics or let-7e-5p inhibitor was transfected to the Schwann cells and NGF mRNA and protein expression was measured. As shown in Fig. 7B and 7C, NGF mRNA level showed no significant change after let-7e-5p mimics or inhibitor transfection but NGF protein level did exhibited significant decrease after cells being transfected with let-7e-5p mimics and increased after being transfected with inhibitor. ELISA assay was used to determine the level of NGF secretion from Schwann cells. The effects of NGF-specific siRNA, let-7e-5p mimics or let-7e-5p inhibitor were compared. It was found that let-7e-5p mimics and NGF siRNA reduced 
and let-7e-5p inhibitor elevated NGF secretion (Fig. 7D). These results implied that let-7e-5p did not alter NGF mRNA stability but participated in the control of NGF protein translation efficiency.

The effects of let-7 miRNA on axon regeneration was investigated by addition of let-7e-5p mimics or let$7 e-5 p$ inhibitor to co-cultured Schwann cells and DRG neurons. The protein expression of GAP-43 (neurite growth) and neurite length measured on GAP-43-stained DRG cells were assessed and compared between different treatment groups. As shown in Fig. 7E-H, let-7e-5p mimics and NGF siRNA led to decreased GAP-43 protein expression and shorter neurite length, whereas let-7e-5p inhibitor resulted in increased GAP-43 expression and longer neurite growth. These results suggested that changes in let-7e$5 p$ expression was correlated with the phenotype changes in axon regeneration in nerves.

\section{Discussion}

Peripheral nerve injury is associated with many clinical conditions, including single nerve damage (mononeuropathy) caused by trauma and multiple nerve damage (polyneuropathy) caused by diabetic hyperglycemia, chemotherapy drugs and hazardous substances used in industries $[2,37,38]$. N-hexane is an organic solvent used in a wide-range of industrial applications, for example, material adhesion, polymer synthetization, oil extraction and rubber processing and widely used in many developing countries. N-hexane is well-known to cause polyneuropathy affecting both motor and sensory nerves in workers with chronic exposure history [1, 2]. Although the peripheral nerves reserve certain regenerative abilities, the regrowth of a damaged nerve would hardly be ideal to achieve complete functional recovery [39]. Currently, the gold standard method for peripheral nerve injury remains to be autologous nerve grafting but is applicable mostly for the mononeuropathy and unsuitable for polyneuropathy, and need to sacrifice a healthy functioning nerve. Therefore, novel therapeutical approaches remained urgently needed for peripheral neuropathies.

BMSCs, adult stem cells derived from bone marrow of patients themselves, are easy to obtain, selftolerated and ethical when being used for autologous transplantation. BMSC transplantation has been regarded an encouraging novel approach in research of peripheral nerve injury treatment [6]. In our earlier study, transplantation with BMSCs has been found to alleviate motor function loss in a rat model of HDinduced polyneuropathy[32]. In another study, BMSCs were found capable of stimulating the NGF-TrkA signaling pathway to promote axon repair [19]. The present study used several quantifiable indexes, including gait score, distal latency and motor nerve conduction velocity to follow-up HD-intoxicated rats receiving BMSC transplantation. Our results confirmed that BMSCs could remarkably improve axon regeneration in rats with HD-caused peripheral neuropathy. In fact, a single dose of BMSCs transplantation was found to introduce long-lasting neuroregenerative effect and the rats demonstrated progressive functional recovery over a period of 5 weeks. Mechanistically, BMSCs were shown to activate the NGF-TrkA-PI3K-Akt-mTOR-CREB pathway, in which the neurotrophin NGF played an indispensable role for BMSCs' neuroregenerative effect. 
Our results were consistent with the findings by others. Hou et al found that bone marrow-derived stromal cells could differentiate into Schwann-cell like cells and promote the regrowth of transected sciatic nerve axons [40]. MSCs transplantation was shown to facilitate the sensory and motor function recovery in paralyzed hind-limbs in rats with chronic spinal cord injury experimentally modelled using ballooninduced compression lesion [41, 42]. Salomone R et al reported that BMSCs could promote axon regeneration in traumatized facial nerve [43]. MSC transplantation has also been shown to improve neurological function recovery in brain damage caused by $\mathrm{CO}$ poisoning in a rat model [44]. Together, the results by us and others reassured the therapeutical potential of MSCs/BMSCs by promoting neuroregeneration in the peripheral and central nervous system. In addition, our study was among the very few studies investigating the efficiency of BMSCs to treat polyneuropathies caused by occupational hazards such as N-hexane.

Because the NGF-TrkA signaling axis was found to play a pivotal role in BMSC-mediated axon regeneration, it is important to understand the possible cellular resources of NGF in our experiments. On one hand, BMSCs could be a direct resource of NGF[45, 46]. In fact, our ELISA analysis confirmed that NGF was present in BMSC-derived CM in a concentration of $\sim 60 \mathrm{pg} / \mathrm{ml}$ (supplemental Fig. 2). On the other hand, the present study revealed another indirect mechanism that BMSCs decreased the expression of let-7 miRNAs and the latter bond to the 3'UTR of NGF mRNA and negatively affected NGF protein translation in Schwann cells (Figs. 6 and 7). Our microarray data, qPCR analysis and in vitro experiments found that: 1. BMSCs caused concordant changes in let-7 miRNA expression and NGF protein expression in sciatic nerves and Schwann cells; 2 . The addition of let-7 miRNA mimics or inhibitors to Schwann cells caused significant change of NGF expression and affected the neurite growth in DRG neurons. The regulatory role of let-7 miRNAs in NGF expression in Schwann cells has also been observed by other investigators $[35,47]$. Collectively, we propose a dual-way mechanism that there could exist an extrinsic pathway in which BMSCs exogenously secret NGF to stimulate axon regeneration, and an intrinsic pathway in which BMSCs regulate NGF expression in Schwann cells via let-7 miRNAs.

MicroRNAs (miRNAs) are a class of non-coding single-strand RNAs that regulated gene expression by promoting degradation or inhibiting protein translation of target mRNAs. The present study focused on understanding the role of let-7 family miRNAs in axonal regeneration by regulating the abundance of neurokine NGF. Similarly, Li et al also observed that the regulatory effect of let-7 on NGF expression and proved that let-7 miRNAs could bond directly to the 3'untranslated region of NGF mRNA[35]. Furthermore, in addition to let-7, our microarray data also revealed another 41 other miRNAs significantly upregulated after HD intoxication (HD vs control) and downregulated after BMSC treatment (HD + BMSC vs HD) and 28 miRNAs significantly downregulated in response to HD and upregulated to BMSC (supplemental Fig. 3A). Among these miRNAs, there are certain interesting candidate miRNAs also likely participating in the regulation of axon regeneration (supplemental Fig. 3B listed their predicted target genes involved in axonogenesis (mirdb.org)). For example, miR-344b-1-3p level was found higher in HD-exposed nerves and lowered after addition of BMSC. miR-344b-1-3p is predicted to specifically target on neurotrophin 3 . To understand the changes of those neurotrophins other than NGF, qPCR experiments were carried out and revealed the expressional differences in BDNF, neurotrophin 3, neurotrophin 4 when comparing among 
control, HD and HD + BMSC groups (supplemental Fig. 3C). Besides, miR-493-5p was found significantly hyperexpressed in the forebrains of rats with epilepsy [48] and its level was also dramatically altered in our experiment. miR-582-3p was associated with NOS3 (endothelial type) expression and neuroinflammation during cerebral ischemia reperfusion injury[47] and it was found upregulated in the nerves of HD group animals and downregulated after BMSC transplantation. Although in the present study, it is not possible for us to mechanistically elaborate the effects of all these miRNAs, these results may inspire other investigators to study in the future.

\section{Conclusions}

In conclusion, the present study revealed that BMSCs transplantation could improve axon regeneration and motor function recovery after HD-induced neurotoxication via interfering with let-7 miRNA's regulation of NGF expression and/or directly secreting exogenous NGF for neuron nourishment. A dual-way mechanistical hypothesis was proposed based on our observations. Our results might hopefully encourage more investigations evaluating BMSC transplantation or application of BMSC-derived acellular products in polyneuropathy treatment and exploring novel mechanisms during this process.

\section{Abbreviations}

PN: peripheral neuropathy; HD: hexanedine; MSC: mesenchymal stem cell; BMSC: bone marrow-derived mesenchymal stem cell; BMSC-CM: BMSC-derived conditional medium; NGF: nerve growth factor; TrkA: tyrosine kinase receptor type 1; DRG: dorsal root ganglia; MCV: motor nerve conduction velocity; SEM: Scanning Electron microscope;

\section{Declarations}

Ethics approval: The animal experiments were approved by the Ethical Committee of Dalian Medical University, China (approval number: CXK (Liao) 2015-2003) and performed following the Institutional Animal Care and Use Committees (IACUC) of Dalian Medical University and following the international guidelines for the animal care and use (ARRIVE guidelines and NIH guidelines).

Consent for publication: Not applicable.

Availability of data and materials: All data generated or analyzed during this study are included in this published article. Further requests are welcomed and please contact the corresponding authors.

Competing interests: The authors declare that they have no competing interests.

Funding: This work was supported by the grants from the National Natural Science Foundation of China (81273038 and 81773402) and the Dalian Municipal Science and Technology Plan Project (2013E15SF163). 
Authors' Contributions: Project administration and study design: Piao F., Wang R. Gong G., Zuo E. and Hu S.; Experiment performing: Zuo E., Hu S., Zhang C., Shi X., Yang Z. and Li S.; Data analysis and Figure preparation: Zuo E., Zhang C., Li S., Bai Q.; Manuscript preparation and proof-reading: Zuo E., Gong G., Wang R. and Piao F. All authors read and approved the final manuscript.

Acknowledgements: We thank Mr Zewen Qiu (Experimental Animal Center, Dalian Medical University) for assisting with animal experiments. We thank Ms Shuxian Qu (Electron microscope laboratory, Dalian Medical University) for assisting with SEM works.

Conflicts of Interest: The authors declare no conflicts of interests.

\section{References}

1. Mckee RH, Adenuga MD, Carrillo JC. Characterization of the toxicological hazards of hydrocarbon solvents. Crit Rev Toxicol. 2015 Apr;45(4):273-365.

2. Kutlu G, Gomceli YB, Sonmez T, Inan LE. Peripheral neuropathy and visual evoked potential changes in workers exposed to n-hexane. J Clin Neurosci. 2009;16(10):1296-9.

3. Chang YC. Neurotoxic effects of n-hexane on the human central nervous system: evoked potential abnormalities in n-hexane polyneuropathy. J Neurol Neurosurg Psychiatry. 1987;50:269-74.

4. Graham DG, Amarnath V, Valentine WM, Pyle SJ, Anthony DC. Pathogenetic studies of hexane and carbon disulfide neurotoxicity. Crit Rev Toxicol. 1995;25:91-112.

5. Llorens J. Toxic neurofilamentous axonopathies-accumulation of neurofilaments and axonal degeneration. J Int Med. 2013;273:478-89.

6. Shariati A, Nemati R, Sadeghipour Y, Yaghoubi Y, Baghbani R, Javidi K, Zamani M, Hassanzadeh A. Mesenchymal stromal cells (MSCs) for neurodegenerative disease: A promising frontier. Eur J Cell Biol. 2020 Aug;99(6):151097.

7. Lim CY, Han JI, Kim SG, Lee CM, Park HM. Evaluation of autologous bone marrow-derived mesenchymal stem cells on renal regeneration after experimentally induced acute kidney injury in dogs. Am J Vet Res. 2016;77:208-17.

8. Ankeny DP, McTigue DM, Jakeman LB. Bone marrow transplants provide tissue protection and directional guidance for axons after contusive spinal cord injury in rats. Exp Neurol. 2004;190:17-31.

9. Chopp M, Zhang XH, Li Y, Wang L, Chen J, Lu D, Lu M, Rosenblum M. (2000). Spinal cord injury in rat: treatment with bone marrow stromal cell transplantation. Neuroreport. 11, 3001-3005.

10. Neuhuber B, Timothy Himes B, Shumsky JS, Gallo G, Fischer I. Axon growth and recovery of function supported by human bone marrow stromal cells in the injured spinal cord exhibit donor variations. Brain Res. 2005;1035:73-85.

11. Zeng X, Zeng YS, Ma YH, Lu LY, Du BL, Zhang W, Li Y, Chan WY. Bone marrow mesenchymal stem cells in a three-dimensional gelatin sponge scaffold attenuate inflammation, promote angiogenesis, and reduce cavity formation in experimental spinal cord injury. Cell Transplant. 2011;20:1881-99. 
12. Mesentier-Louro LA, Zaverucha-do-Valle $C$, da Silva-Junior AJ, Nascimento-Dos-Santos G, Gubert F, de Figueirêdo AB, Torres AL, Paredes BD, Teixeira C, Tovar-Moll F, et al. Distribution of mesenchymal stem cells and effects on neuronal survival and axon regeneration after optic nerve crush and cell therapy. PloS One. 2014;9:e110722.

13. Mesentier-Louro LA, Zaverucha-do-Valle C, Rosado-de-Castro PH, Silva-Junior AJ, Pimentel-Coelho PM, Mendez-Otero R, Santiago MF. (2016). Bone Marrow-Derived Cells as a Therapeutic Approach to Optic Nerve Diseases. Stem. Cells. Int. 2016, 5078619.

14. Zaverucha-do-Valle C, Gubert F, Bargas-Rega M, Coronel JL, Mesentier-Louro LA, Mencalha A, Abdelhay E, Santiago MF, Mendez-Otero R. Bone marrow mononuclear cells increase retinal ganglion cell survival and axon regeneration in the adult rat. Cell Transplant. 2011;20:391-406.

15. Sowa Y, Kishida T, Imura T, Numajiri T, Nishino K, Tabata Y, Mazda O. (2016). Adipose-Derived Stem Cells Promote Peripheral Nerve Regeneration In Vivo without Differentiation into Schwann-Like Lineage. Plast. Reconstr. Surg. 137, 318e-330e.

16. Cao J, Xiao Z, Jin W, Chen B, Meng D, Ding W, Han S, Hou X, Zhu T, Yuan B, et al. Induction of rat facial nerve regeneration by functional collagen scaffolds. Biomaterials. 2013;34:1302-10.

17. Madduri S, Gander B. Growth factor delivery systems and repair strategies for damaged peripheral nerves. J Control Release. 2012;161:274-82.

18. Sefati N, Norouzian M, Abbaszadeh HA, Abdollahifar MA, Amini A, Bagheri M, Aryan A, Fathabady F, F. Effects of Bone Marrow Mesenchymal Stem Cells-Conditioned Medium on Tibial Partial Osteotomy Model of Fracture Healing in Hypothyroidism Rats. Iran Biomed J. 2018;22:90-8.

19. Zhang X, Kong Y, Sun Y, Qian Z, Gao C, Shi X, Li S, Piao Y, Piao F. Bone marrow mesenchymal stem cells conditioned medium protects VSC4.1 cells against 2,5-hexanedione-induced autophagy via NGF-PI3K/Akt/mTOR signaling pathway. Brain Res. 2018;1696:1-9.

20. Madduri S, Papaloïzos M, Gander B. Synergistic effect of GDNF and NGF on axonal branching and elongation in vitro. Neurosci Res. 2009;65:88-97.

21. Wang Q, Sun G, Gao C, Feng L, Zhang Y, Hao J, Zuo E, Zhang C, Li S, Piao F. Bone marrow mesenchymal stem cells attenuate 2,5-hexanedione-induced neuronal apoptosis through a NGF/AKTdependent pathway. Sci Rep. 2016;6:34715.

22. Li ZH, Liao W, Cui XL, Zhao Q, Liu M, Chen YH, Liu TS, Liu NL, Wang F, Yi Y, Shao NS. Intravenous transplantation of allogeneic bone marrow mesenchymal stem cells and its directional migration to the necrotic femoral head. Int J Med Sci. 2011;8:74-83.

23. Lehning EJ, Jortner BS, Fox JH, Arezzo JC, Kitano T, LoPachin RM. gamma-diketone peripheral neuropathy. I. Quality morphometric analyses of axonal atrophy and swelling. Toxicol Appl Pharmacol. 2000;165:127-40.

24. Wang QS, Song F, Zhao X, Hou LY, Xie KQ. Expression changes of apoptotic-related proteins in nerve tissues of rats treated with allyl chloride. Toxicology. 2007;231:58-67.

25. Gilbert SG, Maurissen JP. Assessment of the effects of acrylamide, methylmercury, and 2,5hexanedione on motor functions in mice. J Toxicol Environ Health. 1982;10:31-41. 
26. Takeuchi Y, Ono Y, Hisanaga N, Kitoh J, Sugiura Y. A comparative study on the neurotoxicity of npentane, $\mathrm{n}$-hexane, and $\mathrm{n}$-heptane in the rat. $\mathrm{Br} \mathrm{J}$ Ind Med. 1980;37:241-7.

27. Lehning EJ, Dyer KS, Jortner BS, LoPachin RM. Axonal atrophy is a specific component of 2,5hexanedione peripheral neuropathy. Toxicol Appl Pharmacol. 1995;135:58-66.

28. LoPachin RM, Jortner BS, Reid ML, Das S. gamma-diketone central neuropathy: quantitative morphometric analysis of axons in rat spinal cord white matter regions and nerve roots. Toxicol Appl Pharmacol. 2003;193:29-46.

29. Song F, Yu S, Zhang C, Zhou G, Wang Q, Xie K. The reversibility of neurofilaments decline induced by 2,5-hexanedione in rat nerve tissues. Biochem Pharmacol. 2008;75:737-44.

30. Li SY, Qi Y, Hu SH, Piao FY, Guan H, Wang ZM, Chen RL, Liu S. Mesenchymal stem cells-conditioned medium protects PC12 cells against 2,5-hexanedione-induced apoptosis via inhibiting mitochondriadependent caspase 3 pathway. Toxicol Ind health. 2017;33:107-18.

31. Gulati BR, Kumar R, Mohanty N, Kumar P, Somasundaram RK, Yadav PS. Bone morphogenetic protein-12 induces tenogenic differentiation of mesenchymal stem cells derived from equine amniotic fluid. Cells Tissues Organs. 2013;198:377-89.

32. Wang Q, Sun G, Gao C, Feng L, Zhang Y, Hao J, Zuo E, Zhang C, Li S, Piao F. Bone marrow mesenchymal stem cells attenuate 2,5-hexanedione-induced neuronal apoptosis through a NGF/AKTdependent pathway. Sci Rep. 2016;6:34715.

33. Cantinieaux D, Quertainmont R, Blacher S, Rossi L, Wanet T, Noël A, Brook G, Schoenen J, Franzen R. Conditioned medium from bone marrow-derived mesenchymal stem cells improves recovery after spinal cord injury in rats: an original strategy to avoid cell transplantation. PloS One. 2013;8:e69515.

34. Kim HJ, Lee JH, Kim SH. Therapeutic effects of human mesenchymal stem cells on traumatic brain injury in rats: secretion of neurotrophic factors and inhibition of apoptosis. J Neurotrauma. 2010;27:131-8.

35. Li S, Wang X, Gu Y, Chen C, Wang Y, Liu J, Hu W, Yu B, Wang Y, Ding F, et al. Let-7 microRNAs regenerate peripheral nerve regeneration by targeting nerve growth factor. Mol Ther. 2015;23:423-33.

36. Tosaki T, Kamiya H, Yasuda Y, Naruse K, Kato K, Kozakae M, Nakamura N, Shibata T, Hamada Y, Nakashima E, et al. Reduced NGF secretion by Schwann cells under the high glucose condition decreases neurite outgrowth of DRG neurons. Exp Neurol. 2008;213:381-7.

37. Staff NP, Grisold A, Grisold W, Windebank AJ. Chemotherapy-induced peripheral. neuropathy. A current review. Ann Neurol. 2017 Jun;81(6):772-81.

38. Zilliox LA. Diabetes and Peripheral Nerve Disease. Clin Geriatr Med. 2021 May;37(2):253-67.

39. Richner M, Ulrichsen M, Elmegaard SL, Dieu R, Pallesen LT, Vaegter CB. Peripheral Nerve Injury Modulates Neurotrophin Signaling in the Peripheral and Central Nervous System. Mol Neurobiol. 2014;50(3):945-70.

40. Hou SY, Zhang HY, Quan DP, Liu XL, Zhu JK. (2006). Tissue-engineered peripheral nerve grafting by differentiated bone marrow stromal cells. Neurosci. 140, 101-110. 
41. Ohta M, Suzuki Y, Noda T, Ejiri Y, Dezawa M, Kataoka K, Chou H, Ishikawa N, Matsumoto N, Iwashita $Y$, et al. Bone marrow stromal cells infused into the cerebrospinal fluid promote functional recovery of the injured rat spinal cord with reduced cavity formation. Exp Neurol. 2004;187:266-78.

42. Hejcl A, Sedý J, Kapcalová M, Toro DA, Amemori T, Lesný P, Likavcanová-Mašínová K, Krumbholcová E, Prádný M, Michálek J, et al. HPMA-RGD hydrogels seeded with mesenchymal stem cells improve functional outcome in chronic spinal cord injury. Stem Cells Dev. 2010;19:1535-46.

43. Salomone R, Bento RF, Costa HJ, Azzi-Nogueira D, Ovando PC, Da-Silva CF, Zanatta DB, Strauss BE, Haddad LA. Bone marrow stem cells in facial nerve regeneration from isolated stumps. Muscle Nerve. 2013;48:423-9.

44. Jiang G, Gao J, Xu Y, Ma Y, Jiang G, Yan W. Structural and functional improvement of injured brain after severe acute carbon monoxide poisoning by stem cell-based therapy in rats. Crit Care Med. 2009;37:1416-22.

45. Crigler L, Robey RC, Asawachaicharn A, et al. Human mesenchymal stem cell subpopulations express a variety of neuro-regulatory molecules and promote neuronal cell survival and neuritogenesis. Exp Neurol. 2006;19:54-64.

46. Villatoro AJ, Alcoholado C, Martin-Astorga MC, et al. Comparative analysis and characterization of soluble factors and exosomes from cultured adipose tissue and bone marrow mesenchymal stem cells in canine species. Vet Immunol Immunopathol. 2019;20:6-15.

47. Zhang Y, Zhang Y. IncRNA ZFAS1 Improves Neuronal Injury and Inhibits Inflammation, Oxidative Stress, and Apoptosis by Sponging miR-582 and Upregulating NOS3 Expression in Cerebral Ischemia/Reperfusion Injury. Inflammation. 2020 Aug;43(4):1337-1350.

48. Gitaí DLG, Dos Santos YDR, Upadhya R, Kodali M, Madhu LN, Shetty AK. Extracellular Vesicles in the Forebrain Display Reduced miR-346 and miR-331-3p in a Rat Model of Chronic Temporal Lobe Epilepsy. Mol Neurobiol. 2020 Mar;57(3):1674-87.

\section{Figures}



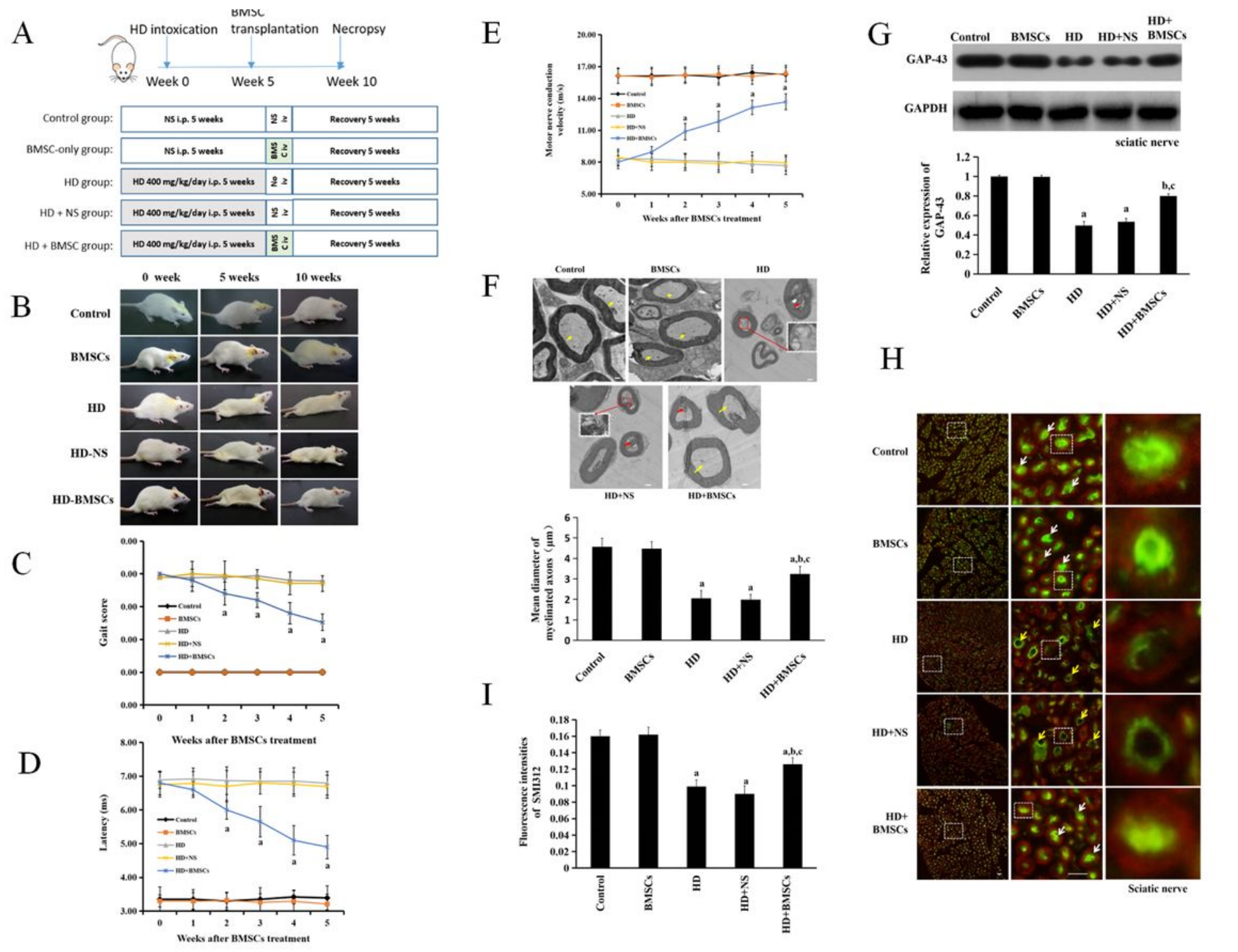

\section{Figure 1}

Axon degeneration caused by HD intoxication and axon regeneration promoted by BMSC transplantation in a rat model. (A) Schematic diagram illustrating the experimental design treating SD rats with HD $(400 \mathrm{mg} / \mathrm{kg} /$ day, ip, for 5 weeks) +/- BMSC transplantation $(5 \times 107 \mathrm{cell} / \mathrm{s} / \mathrm{kg}$ body weight, in a bolus injection via tail vein). The animals were grouped as: Control group (NS i.p. + NS i.v.); BMSC group (NS i.p. + BMSC i.v.); HD group (HD i.p. only); HD + NS group (HD i.p. + NS i.v.); HD + BMSC group (HD i.p. + BMSC i.v.). Animal number was 10 per group. (B) The behavior feature of rats receiving HD intoxication with or without BMSC transplantation. Images were captured by the end of week 10. (C) - (E): The curves showing the changes in gait score, distal latency and motor nerve conduction velocity of rats in different HD +/- BMSC treatment groups over a period of 10 weeks. (F) The ultrastructure of sciatic nerves of rats in different $\mathrm{HD}+/$ - BMSC treatment groups under electron microscopy. The bar graph compares the mean diameters of myelinated axons in these sciatic nerves. (G) Western blot results showing GAP-43 protein levels in sciatic nerves of the aforementioned animals. $(H)$ Representative images of immunofluorescent co-staining of MBP (myelin-specific marker) and SMI312 (axon-specific marker) in sciatic nerves of the aforementioned animals. The fluorescent intensities were quantified and shown besides. Data were 
expressed as mean \pm standard error of the mean, ap $<0.05$ versus control, $b p<0.05$ versus HD group, $c p<$ 0.05 versus HD + NS group. Scale bar, $0.5 \mu \mathrm{m}$.

A

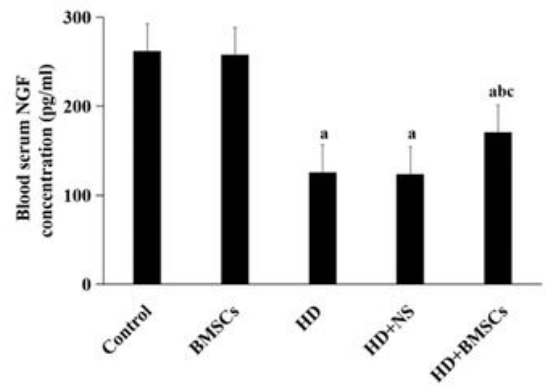

$\mathrm{C}$

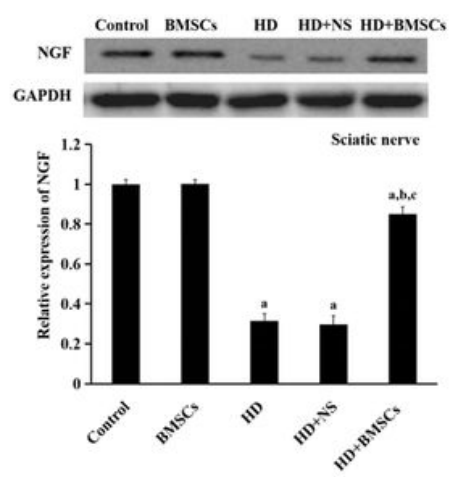

$\mathrm{E}$

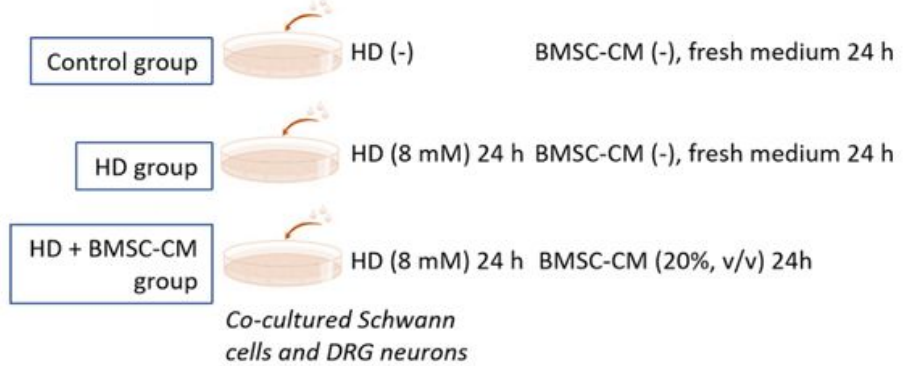

$\mathrm{B}$

$\mathrm{D}$
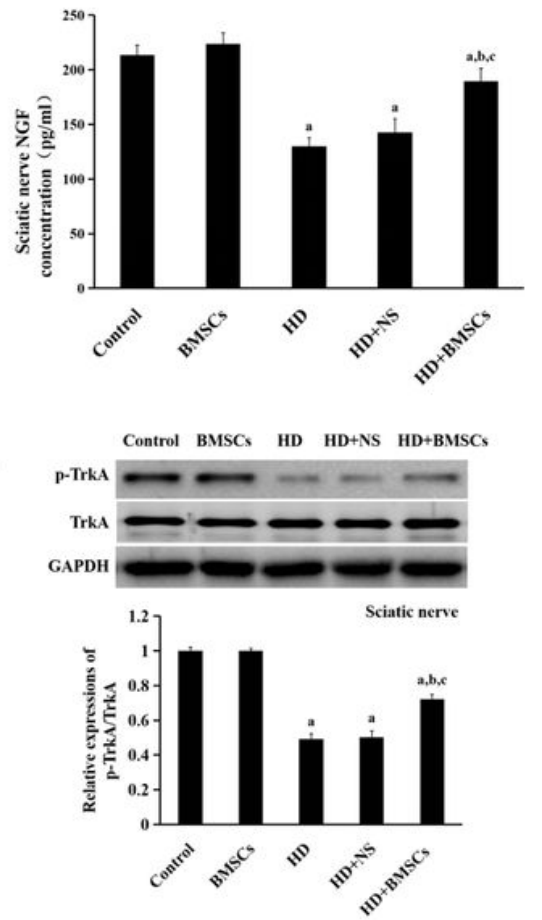

G
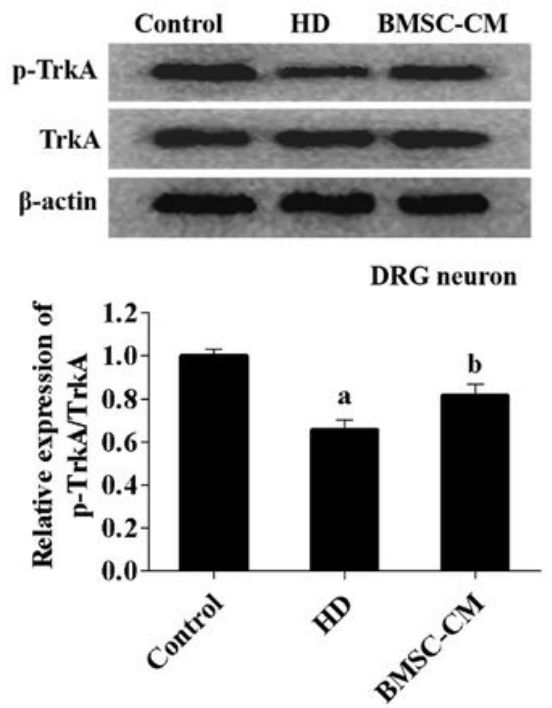

Figure 2

NGF level and TrkA activation significantly changed after BMSC transplantation. (A) - (B) NGF concentration in serum and sciatic nerves, respectively, in HD/BMSC treated animals using an ELISA assay. (C) - (D) Western blot results showing NGF protein expression and TrkA activation ( $p$-TrkA) in sciatic nerves of HD/BMSC treated animals. (E) Schematic diagram showing cell culture experiment treating co-cultured Schwann cells and DRG cells with HD and with or without BMSC-CM. Control group: no treatment. HD group: cells were exposed to $8 \mathrm{mM}$ HD for $24 \mathrm{~h}$ and then replaced with fresh medium for another 24h growth. HD + BMSC group: cells were exposed to 8mM HD for 24h and then replaced with BMSC-CM (20\% v/v)-containing fresh medium for another 24h. ( $\mathrm{G}$ and $\mathrm{H}$ ) Western blot results showing NGF protein expression and TrkA activation ( $p$-TrkA) in HD/BMSC treated co-cultured Schwann and DRG cells. Data were expressed as mean \pm standard error of the mean, ap $<0.05$ versus control, $b p<0.05$ versus HD group, $\mathrm{cp}<0.05$ versus HD + NS group. 
A

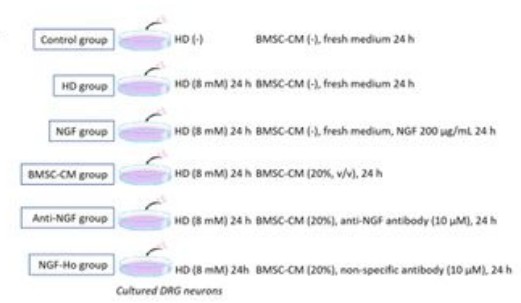

E
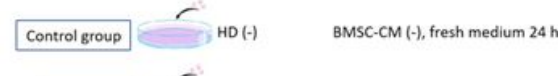

HD group $\longrightarrow$ HD $(8 \mathrm{mM}) 24 \mathrm{~h}$ BMSC.CM (-), fresh medium $24 \mathrm{~h}$

BMSC.CM group $\quad$ HD $(8 \mathrm{mM}) 24 \mathrm{~h} \mathrm{BMSC-CM}(20 \%, \mathrm{v} / \mathrm{v}), 24 \mathrm{~h}$

K252a group $\sim$ HD (8 mM) 24h BMSC-CM (20\%), K252a (200 mM), $24 \mathrm{~h}$ Cultured DRG neurons

C
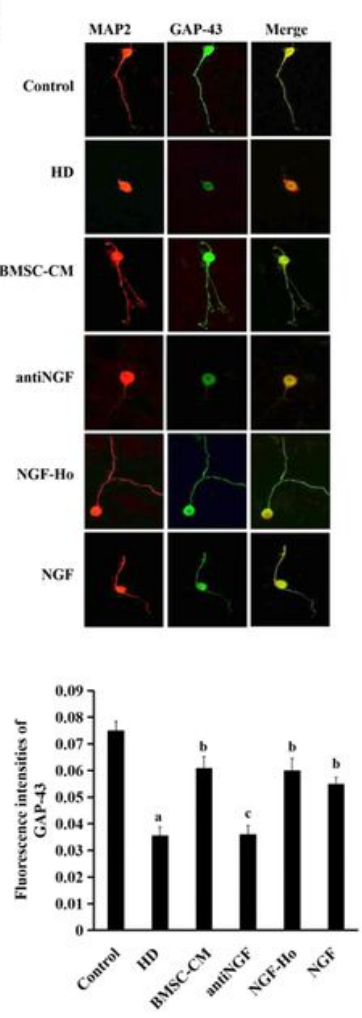

B

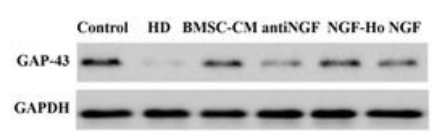

$\mathrm{D}$
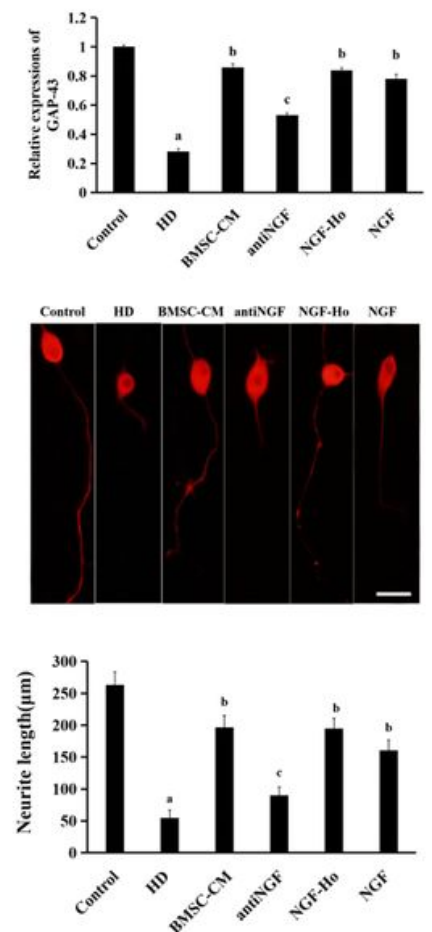

F

Control HD BMSC-CM K252

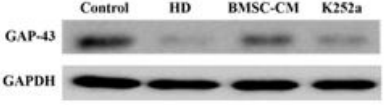

G
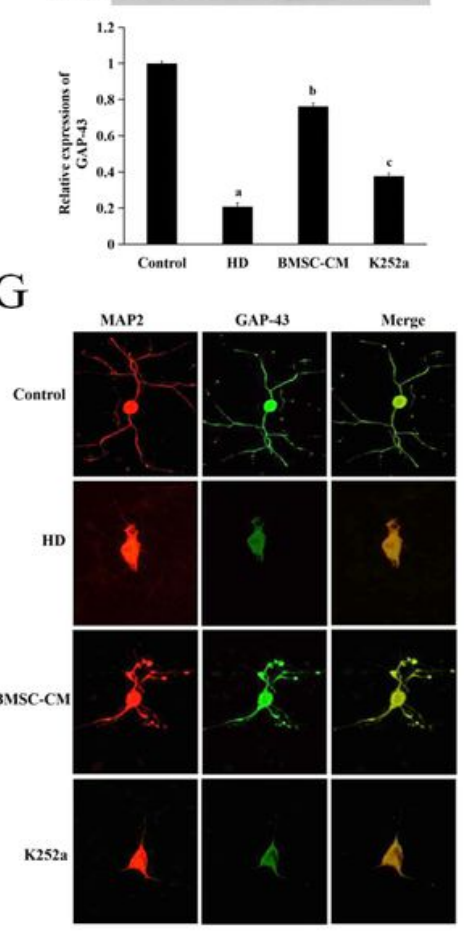

$\mathrm{H}$
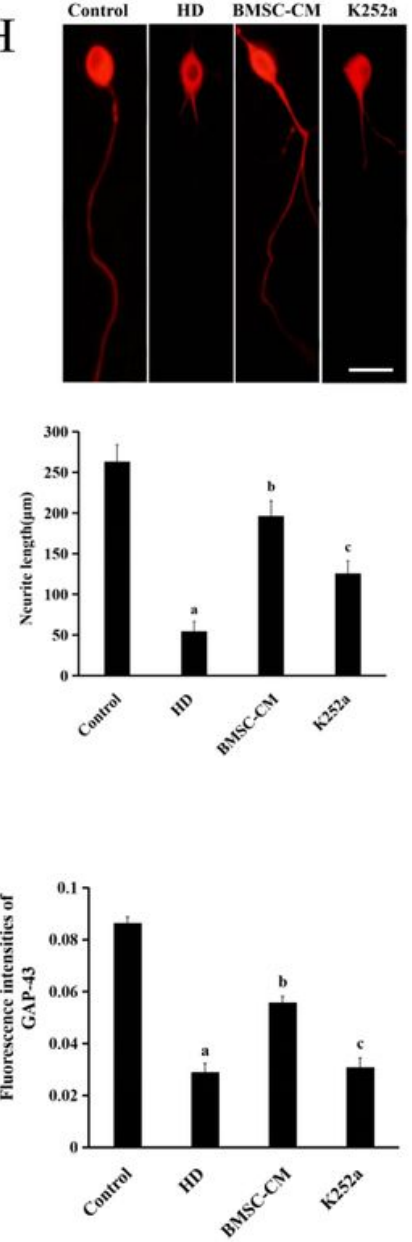

\section{Figure 3}

NGF was a key mediator for BMSC induced axonal regeneration in vitro. (A) Schematic diagram showing cell culture experiment treating cultured DRG neurons with HD (8mM) +/- BMSC-CM (20\% v/v) +/recombinant NGF (200ug/ml) or anti-NGF antibody (10uM) or non-specific immunoglobulin (10uM). Control group: no treatment; HD group: cells were treated only with HD for 24h and fresh medium for another 24h; NGF group: cells were treated with HD for 24h and recombinant NGF for another 24h; BMSCCM group: cells were treated with HD for $24 \mathrm{~h}$ and BMSC-CM for another $24 \mathrm{~h}$; anti-NGF group: cells were treated with HD for 24h and BMSC-CM together with anti-NGF antibody for another 24h. NGF-Ho group: cells were treated with HD for $24 \mathrm{~h}$ and BMSC-CM together with non-specific antibody for another $24 \mathrm{~h}$. (B) Western blot results showing GAP-43 protein levels in sciatic nerves of treatment groups described in (A). (C) Representative images of immunofluorescent co-staining of MAP2 (neuron-specific marker) and GAP43 in sciatic nerves of treatment groups described in (A). The fluorescent intensities were quantified and shown besides. (D) The axon lengths of DRGs were measured in cells of different groups (C). (E) Schematic diagram showing cell culture experiment treating cultured DRG neurons with HD (8mM) +/BMSC-CM (20\% v/v) +/- NGF inhibitor K252a (200mM). Control group, HD group and BMSC-CM group 
were as described above. K252a group: cells were treated with HD for 24h and BMSC-CM together with K252a for another 24h. (F) Western blot results showing GAP-43 levels in treated cells described in (E). (G) Immunofluorescent co-staining of MAP2 and GAP43 in treated cells described in (E). (H) The axon lengths of DRGs measured in treated cells described in (E). Data were expressed as mean \pm standard error of the mean, ap $<0.05$ versus control, bp $<0.05$ versus HD group, $c p<0.05$ versus HD+BMSC-CM group. Scale bar, $50 \mu \mathrm{m}$.

A
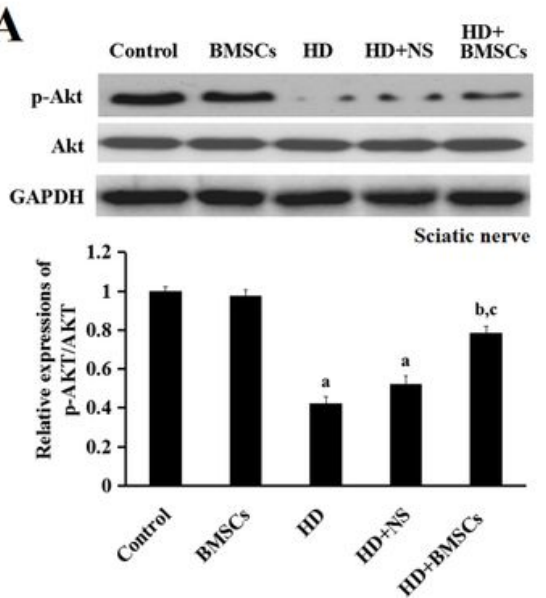

D
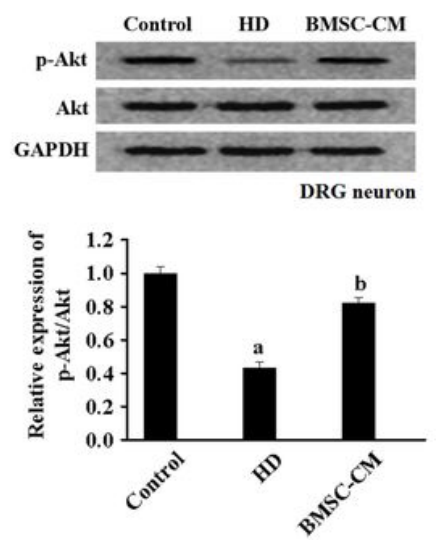

B
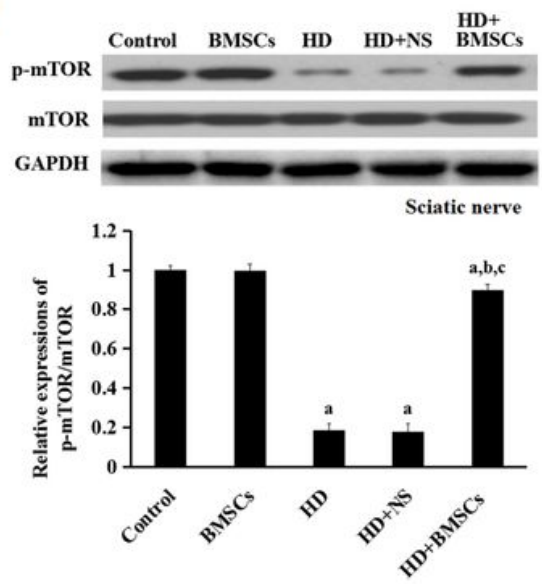

$\mathbf{E}$
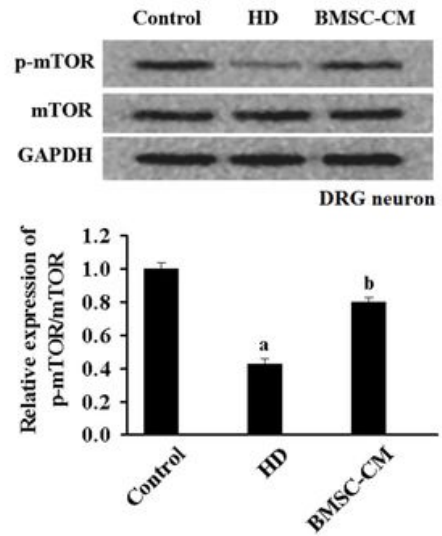

C
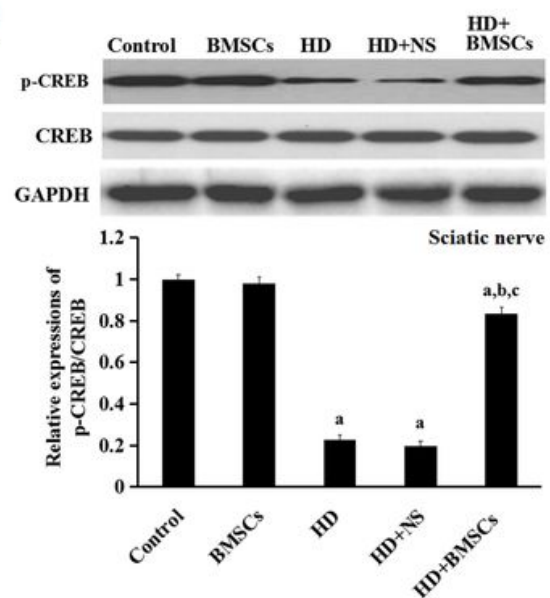

$\mathbf{F}$
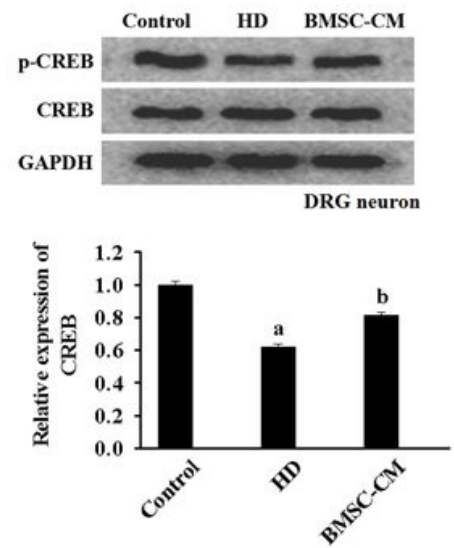

\section{Figure 4}

The Akt-mTOR-CREB signaling molecules downstream to NGF-TrkA activation were changed in HD +/BMSC/BMSC-CM treated sciatic nerves and cultured DRG neurons. (A)-(C) Western blot results showing changed protein expression of Akt, p-Akt, mTOR, p-mTOR, CREB and p-CREB in sciatic nerves of rats receiving HD treatment with or without BMSCs transplantation. (D)-(F) Western blot results showing changed protein expression of Akt, p-Akt, mTOR, p-mTOR, CREB and p-CREB in cultured DRG neurons receiving HD treatment with or without BMSC-CM treatment. Data were expressed as mean \pm standard error of the mean, $a p<0.05$ versus control, $b p<0.05$ versus $H D$ group, $c p<0.05$ versus $H D+B M S C$ group. 
A

Control group $\sim$ HD (-) BMSC-CM (-), fresh medium 24h

HD group

BMSC.CM group $\sim$ HD (8mM) 24h BMSC-CM (20\%, v/v), 24h

K252a group

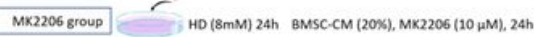

Rapamycin group Cuitured $D R G$ neurons

$\mathrm{C}$

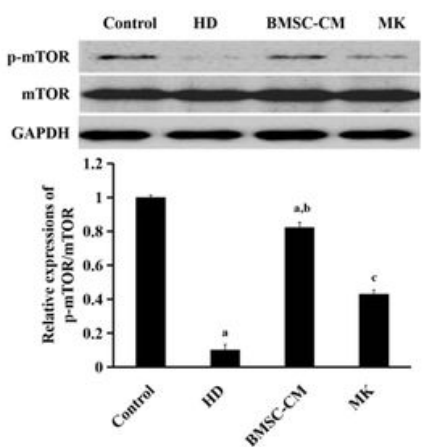

$\mathrm{E}$

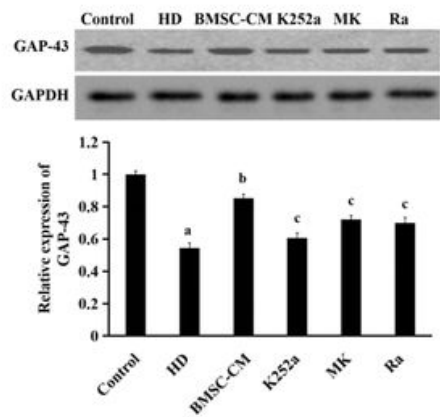

B

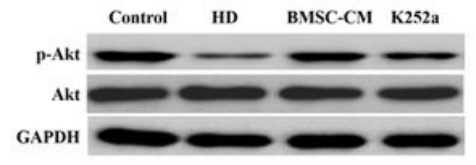

$\mathrm{D}$
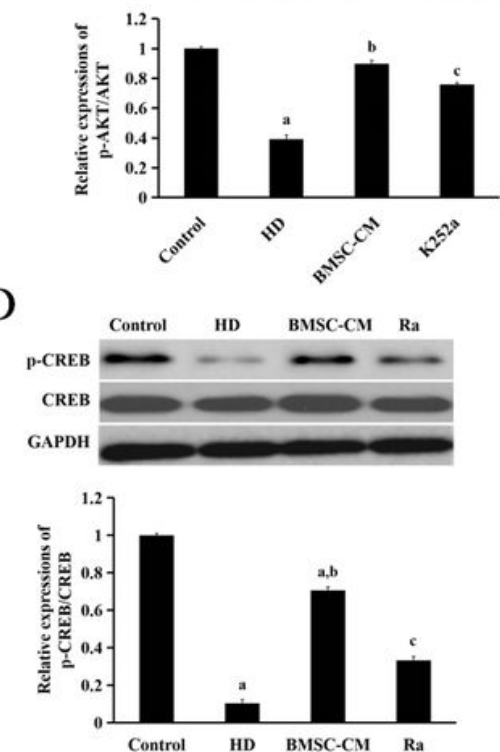

G

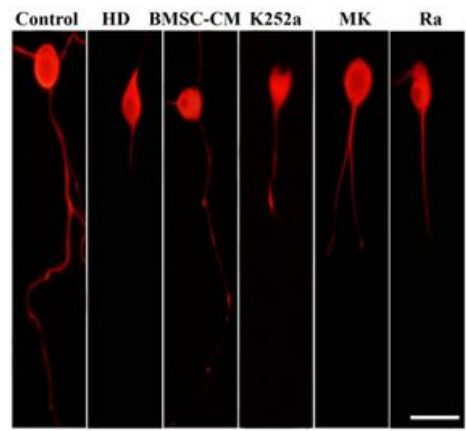

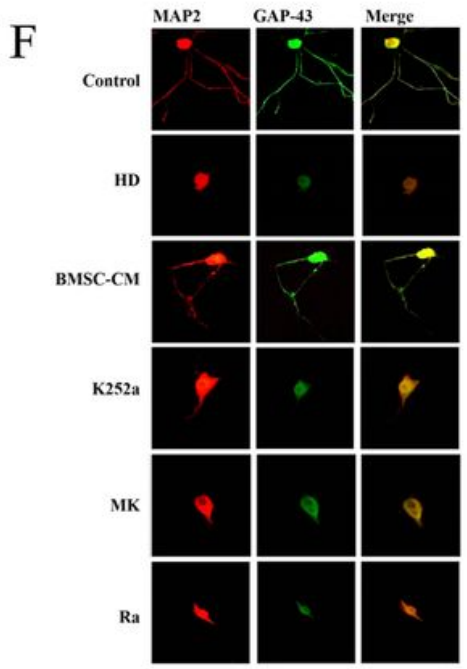
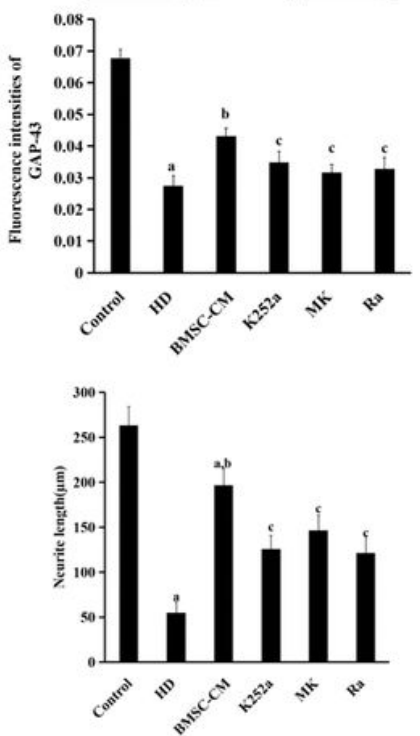

\section{Figure 5}

Blockade of Akt-mTOR-CREB signaling axis resulted in blockade of BMSC induced axonal regeneration in vitro. (A) Schematic diagram showing cell culture experiment treating cultured DRG neurons with HD $(8 \mathrm{mM})+/-$ BMSC-CM (20\% v/v) +/- NGF inhibitor K252a (200mM). Control group, HD group and BMSC$\mathrm{CM}$ group were as described above. K252a group: cells were treated with HD for 24h and BMSC-CM together with K252a for another 24h. (B)-(D) The protein expression levels of Akt and pAkt after addition of NGF inhibitor K252a, mTOR and p-mTOR after addition of Akt inhibitor MK2206, CREB and pCREB after addition of Rapamycin, respectively, in the cultured DRG neurons receiving HD +/- BMSC treatments. (E) Western blot results showing GAP-43 levels in treated cells described in (A). (F) Immunofluorescent costaining of MAP2 and GAP43 in treated cells described in (A) (G) The axon lengths of DRGs measured in treated cells described in (A). Data were expressed as mean \pm standard error of the mean, ap $<0.05$ versus control, $b p<0.05$ versus HD group, $c p<0.05$ versus HD + BMSC-CM group. Scale bar, $50 \mu \mathrm{m}$. 
A

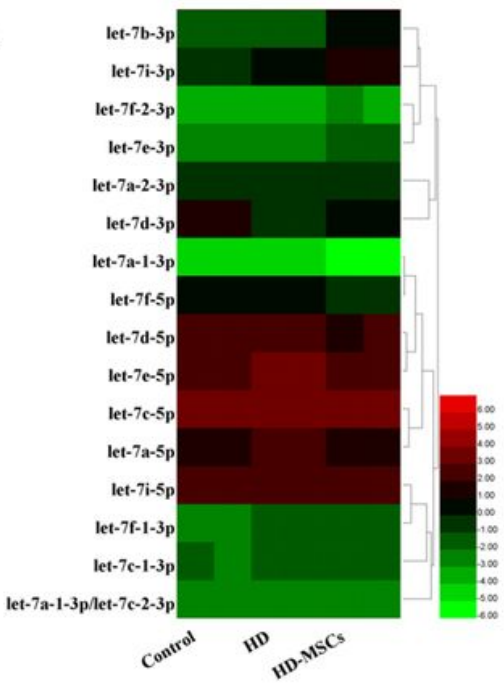

F

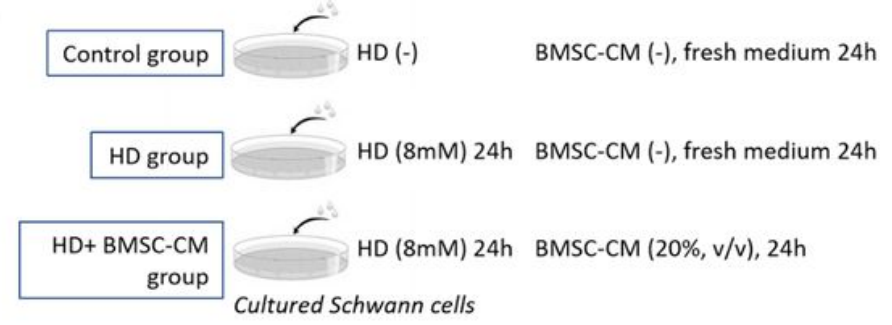

$\mathrm{H}$

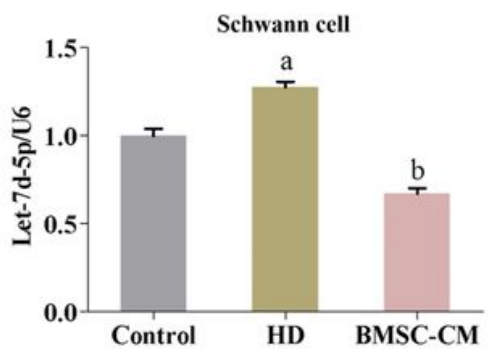

B

$\mathrm{D}$
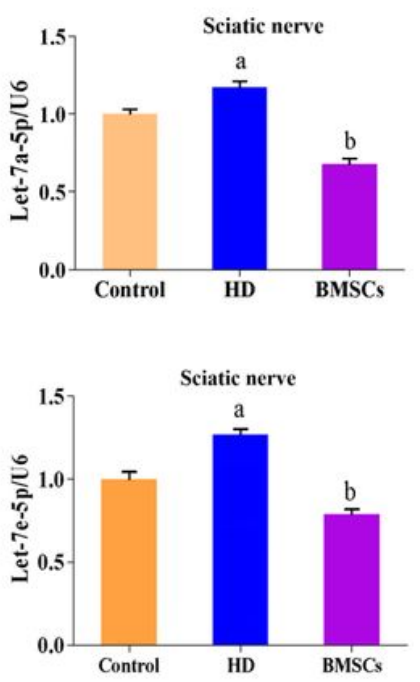

G

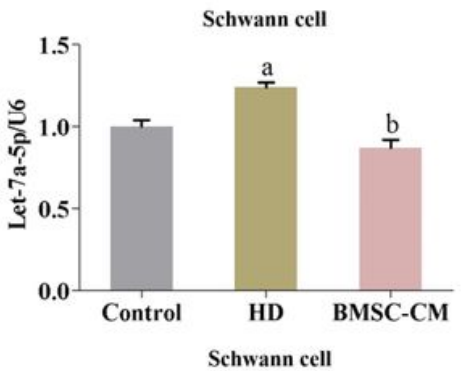

$\mathrm{J}$

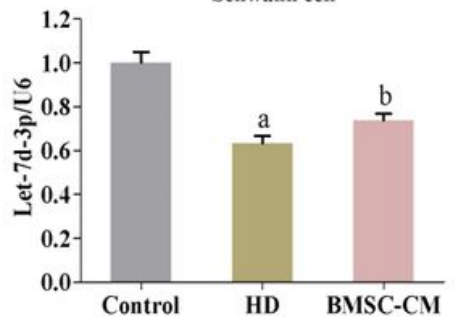

Figure 6

miRNA array profile and RT-PCR validation of let-7 family miRNAs in sciatic nerves of rats receiving HD and BMSCs treatment. (A) Heatmap and clustering from miRNA array showed the expressional changes of let-7 family miRNAs in sciatic nerves of untreated (control), HD-treated (HD) and HD + BMSCs-treated $(H D+B M S C)$ rats. (relative to that of U6) (B)-(E) RT-PCR validation of the changed expression of let-7a-5p, let-7d-5p, let-7e-5p and let-7d-3p miRNAs in sciatic nerves. (F) Schematic diagram showing cell culture experiment treating cultured Schwann cells with HD (8mM) +/- BMSC-CM (20\% v/v), Control group: no treatment; HD group: cells were treated only with $8 \mathrm{mM} \mathrm{HD}$ for $24 \mathrm{~h}$ and fresh medium for another $24 \mathrm{~h} ; \mathrm{HD}$ + BMSC-CM group: cells were exposed to $8 \mathrm{mM} \mathrm{HD}$ for $24 \mathrm{~h}$ and then replaced with BMSC-CM $(20 \% \mathrm{v} / \mathrm{v})$ containing fresh medium for another 24h. (G)-(J) RT-PCR validation of the changed expression of let-7a$5 p$, let-7d-5p, let-7e-5p, and let-7d-3p miRNAs in cultured Schwann cells. Data were expressed as mean \pm standard error of the mean, $a p<0.05$ versus control, $b p<0.05$ versus HD group. 
A

Position 56-63 of NGF 3' UTR mi-let-7e-5p

5 '...CUCCUGGGCCCCUCCCUACCUCA...

| | | | | |

3' UUGAUAUGUUGGAGGAUGGAGU

C

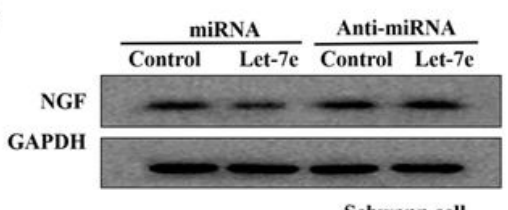

Schwann cell

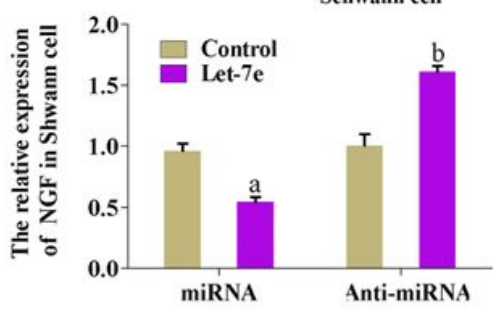

E

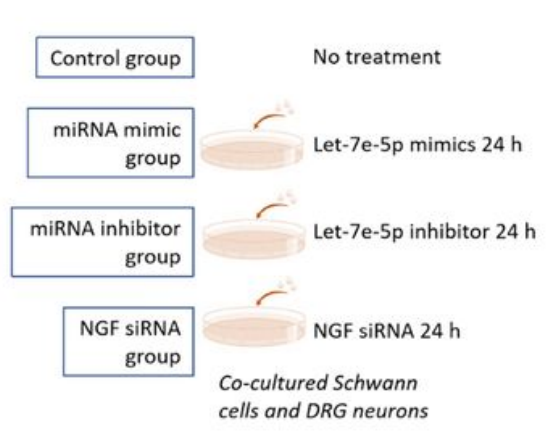

D

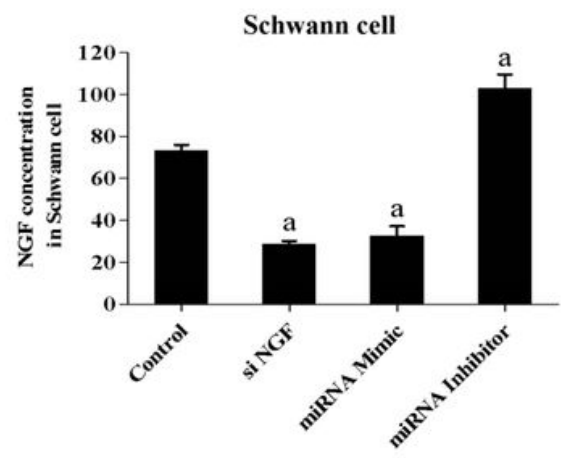

F
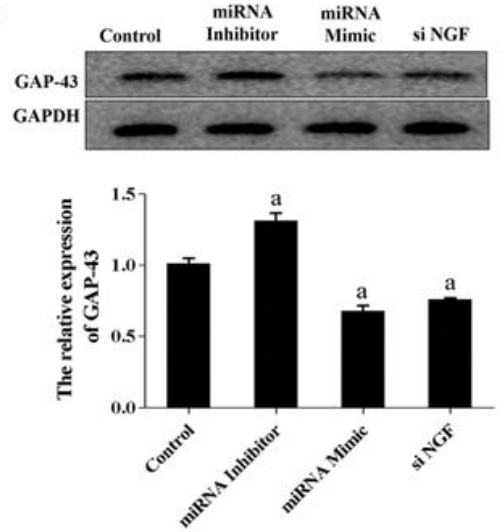

B

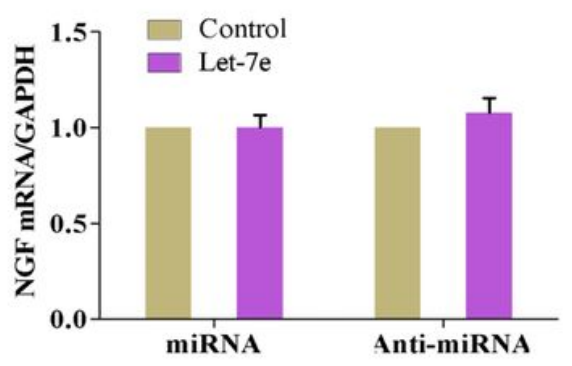

G

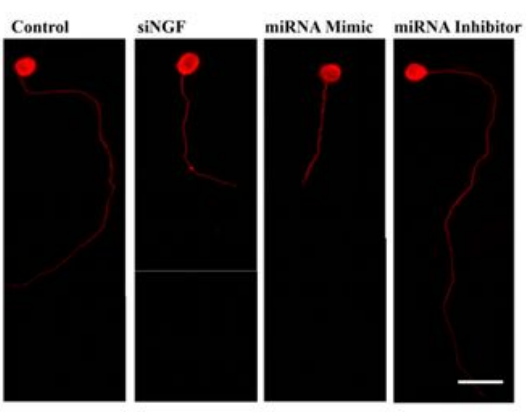

$\mathrm{H}$

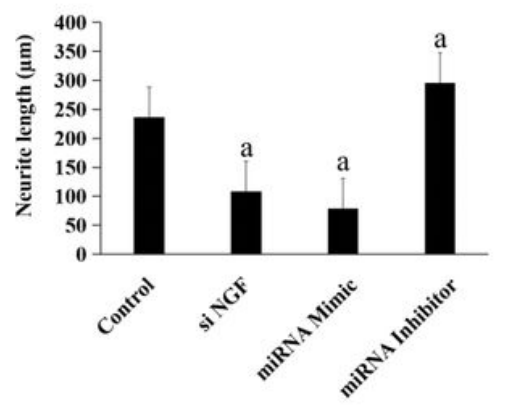

\section{Figure 7}

Inhibition of let-7e-5p, a miRNA suppressing NGF production in Schwann cells, promoted axon regeneration. (A) Sequence alignment of the putative let-7e-5p binding sites on the 3'-untranslated region (3'-UTR) of NGF mRNA. (B)-(C) The mRNA (B) and protein (C) expression levels of NGF were determined in Schwann cells transfected with let-7e-5p mimics or inhibitors, respectively. (D) Schwann cells were transfected with NGF-siRNA, let-7e-5p mimics and let-7e-5p inhibitors and NGF concentration in culture medium was determined using ELISA assay. (E) Schematic diagram showing co-cultured DRG and Schwann cells being treated with NGF-siRNA, let-7e-5p mimics and let-7e-5p inhibitors, respectively. (F) Western blot results showing GAP-43 expression cells treated with NGF-siRNA, let-7e-5p mimics and let7e-5p inhibitors, as described above. (G)-(H) Neurite length (of DRG cells) measurement in GAP-43immunostained cells as described in $(E)$. Data were expressed as mean \pm standard error of the mean, aP< 0.05 versus respective controls. Scale bar, $50 \mu \mathrm{m}$

\section{Supplementary Files}


This is a list of supplementary files associated with this preprint. Click to download.

- SupplementalFig1.jpg

- SupplementalFig2.jpg

- SupplementalFig3.jpeg 